\title{
TAXA DE APLICAÇÃO E USO DE SURFACTANTE SILICONADO NA DEPOSIÇÃO DA PULVERIZAÇÃO E CONTROLE DA FERRUGEM DA SOJA
}

Doi:http://dx.doi.org/10.1590/1809-4430-Eng.Agric.v35n3p 514-527/2015

\section{EVANDRO P. PRADO ${ }^{1}$, CARLOS G. RAETANO ${ }^{2}$, MÁRIO H. F. DO A. DAL POGETTO ${ }^{3}$, SAULO I. DE A. COSTA ${ }^{4}$, RAFAEL DE S. CHRISTOVAM ${ }^{5}$}

RESUMO: A eficiência do controle químico da ferrugem-asiática da soja (FAS), além de outros fatores, está associada ao uso da técnica de aplicação de fungicida mais adequada. O trabalho teve por objetivo avaliar tecnologias de aplicação sobre os depósitos da pulverização e controle da FAS nos anos agrícolas de 2009-2010 e 2010-2011. O delineamento experimental foi em blocos casualizados, e os tratamentos distribuídos no esquema fatorial 3x2 (três taxas de aplicação: 60; 110 e $160 \mathrm{~L} \mathrm{ha}^{-1}$ com e sem adição de surfactante siliconado) sobre os depósitos da pulverização, usando como marcador o corante Azul Brilhante, em quatro repetições (Experimento 1). Para avaliação da severidade da doença, peso de mil grãos (PMG) e produtividade, foi adotado o mesmo delineamento do primeiro experimento, com acréscimo de uma testemunha $(3 \times 2+1)$, utilizando a mistura fungicida epoxiconazole associado com azoxistrobina, com quatro repetições (Experimento 2). As taxas de aplicação e o uso do surfactante não influenciaram os níveis dos depósitos da pulverização nas folhas das partes mediana e inferior da planta. Pulverização com a mistura fungicida na taxa de $160 \mathrm{~L} \mathrm{ha}^{-1}$ proporcionou maior controle da FAS e maior produtividade no ano agrícola de 2010-2011. O controle químico com fungicida, na taxa de aplicação adequada ao estádio de desenvolvimento da planta, torna-se indispensável para a garantia da produtividade na cultura da soja.

PALAVRAS-CHAVE: adjuvantes, depósitos da pulverização, tec nologia de aplicação.

\section{EFFECTS OF SILICONE SURFACTANT AND APPLICATION RATES ON SPRAY DEPOSITION AND SOYBEAN RUST CONTROL}

\begin{abstract}
Chemical control efficiency of Asian soybean rust (ASR), besides other factors, is associated with an appropriate fungicide application technique. This study aimed to evaluate the effect of different application technologies on spray deposition and ASR control in 2009-2010 and 2010-2011 crop seasons. The experimental design was a completely randomized block with four replications in a $3 \times 2$ factorial scheme. Three application rates were tested $\left(60,110\right.$ and $\left.160 \mathrm{~L} \mathrm{ha}^{-1}\right)$ with and without silicone surfactant addition using a Brilliant Blue dye tracer in spray solution to analyze deposition (Experiment 1). For disease severity, thousand grain weight (TGW) and yield were evaluated on the same experimental design as used in Experiment 1 plus a control $(3 \times 2+1)$. It was used the fungicide mixture of azoxystrobin and epoxiconazol in four replications against ASR (Experiment 2). Application rates and surfactant use did not affect spray deposit levels on middle and bottom plant parts. The fungicide mixture at $160 \mathrm{~L} \mathrm{ha}^{-1}$ was more effective for disease control and showed a greater yield in the 2010-2011 crop season. Chemical control with fungicides and application rates proper to the plant growing stage become essential to ensure a good soybean yield.
\end{abstract}

KEYWORDS: adjuvants, spray deposition, application technology

\footnotetext{
${ }^{1}$ Eng $^{\circ}$ A grônomo, Prof. Assistente Doutor, Curso de Engenharia A gronômica, UNESP - Câmpus de Dracena - SP, Fone: (18) 3821 7485, epprado@dracena.unesp.br

${ }^{2}$ Eng $^{\circ}$ A grônomo, Prof. Adjunto, Departamento de Proteção Vegetal, FCA/UNESP/Botucatu - SP, raetano@fca.unesp.br

${ }^{3}$ Eng $^{\circ}$ A grônomo, Doutor em Proteção de Plantas - Dow AgroScience - Pesquisador Sênior, mhfadpogetto@y ahoo.com.br

${ }^{4}$ Eng $^{\circ}$ A grônomo - Doutor em Agricultura - Terracal Alimentos e Bioenergia - Analista Técnico de Experimentos, sauloagro@gmail.com

5 Eng $^{\circ}$ A grônomo, Prof. de A gronomia, FUNEP/Penápolis - SP, srchristovam@bol.com.br

Recebido pelo Conselho Editorial em: 09-9-2013
}

Aprovado pelo Conselho Editorial em: 02-2-2015 


\section{INTRODUÇÃO}

A ferrugem-asiática da soja (FAS), ocasionada pelo fungo Phakopsora pachyrhizi, identificada no Brasil no final da safra de 2000-2001, ainda causa perda significativa aos sojicultores. Os primeiros sintomas da doença iniciam-se nas folhas da parte inferior e multiplicam-se até atingir as folhas da parte superior das plantas (ZHU et al., 2008). Esta ocorrência está relacionada às condições climáticas favoráveis (temperaturas médias menores que $28{ }^{\circ} \mathrm{C}$ e molhamento foliar acima de 10 horas). O período crítico da doença tem-se mostrado na fase reprodutiva da cultura, a partir do florescimento, porém pode ocorrer já nos primeiros estádios de desenvolvimento (FURLAN, 2005).

Embora haja pesquisadores investigando resistência genética de cultivares de soja ao fungo $P$. pachyrhizi (CALVO et al., 2008; WALKER et al., 2011), ainda não estão disponíveis materiais resistentes a todas as raças desse patógeno (GOELLNER et al., 2010), sendo o controle com pulverizações fungicidas a alternativa mais viável na redução de perdas (LEVY, 2005).

Uma das formas de maximizar a eficiência de produtos fitossanitários é a utilização de adjuvantes em caldas de pulverização. O uso de óleos ou de surfactantes, adjuvantes com capacidade de redução da tensão superficial, associados com produtos fitossanitários podem oferecer melhorias na eficácia de pulverizações (ZABKIEWICZ, 2008). Os adjuvantes, a depender do tipo, promovem melhorias na deposição das gotas nas folhas, no espalhamento, na aderência e na penetração dos produtos fitossanitários, sendo assim características importantes em seu modo de ação (RYCKAERT et al., 2007).

A absorção pelas folhas pode ocorrer de forma lenta pela cutícula ou fisicamente pelos estômatos. Estudos demonstraram que líquidos com tensão superficial de $76 \mathrm{mN} \mathrm{m}{ }^{-1}$ não conseguem atravessar os estômatos das folhas e atingir o mesófilo foliar. Soluções contendo surfactante à base de organossilicones são capazes de molhar até mesmo superfícies altamente hidrofóbicas, como Teflon, podendo oferecer um excelente espalhamento nas superfícies foliares. Soluções contendo organossilicones podem apresentar tensão superficial de $22 \mathrm{mN} \mathrm{m}{ }^{-1}$, valor bem abaixo do necessário para que haja absorção via estômato (ZABKIEWICZ, 2008).

IOST \& RAETANO (2010), avaliando a tensão superficial dinâmica e o ângulo de contato de soluções aquosas com surfactantes em superfícies naturais e artificiais, concluíram que níveis de molhamento maiores em alvos naturais foram obtidos por soluções aquosas com menores tensões superficiais e menor ângulo de contato das gotas com a superfície, porém essas propriedades são dependentes das características das espécies vegetais.

Outro aspecto a ser considerado na tecnologia de aplicação para o controle da doença FAS é o tipo de ponta e a taxa de aplicação necessária para obter cobertura eficiente do alvo a ser atingido sem que haja desperdício de produtos por escorrimento. Atualmente, existe tendência de redução da taxa de aplicação (BOLLER \& MACHRY, 2007; CUNHA et al., 2008), pois os volumes praticados na agricultura, em muitos casos, são muito maiores que aqueles necessários para o controle dos agentes nocivos às plantas. A redução da taxa de aplicação requer otimização da tecnologia de aplicação para assegurar a manutenção da eficiência das aplicações (CUNHA \& SILVA JÚNIOR, 2005).

ARMSTRONG-CHO et al. (2008), avaliando o efeito da taxa de aplicação no controle da queima do grão-de-bico causada pelo fungo Ascochyta rabiei, não observaram interação significativa entre taxa de aplicação e partes de amostragem das plantas, sugerindo que o aumento da taxa não muda a proporção de calda retida nas partes inferior, mediana e superior dessa planta. Vale lembrar que o índice de massa foliar da cultura e a maneira como as folhas estão dispostas na planta são fatores importantes a serem considerados neste estudo.

Embora seja grande a importância de uma boa cobertura da calda fungicida, a literatura científica contém poucos estudos referentes à taxa de aplicação mais adequada para cada problema fitos sanitário (ARMSTRONG-CHO et al., 2008). Em decorrência da redução da tensão superficial da calda proporcionada pela utilização de surfactante siliconado, surgiu a hipótese da possibilidade 
da redução da taxa de aplicação sem comprometer a eficiência de controle. Dessa forma, este trabalho teve como objetivos avaliar o efeito de surfactante siliconado e diferentes taxas de aplicação sobre a deposição da calda de pulverização e o controle da FAS.

\section{MATERIAL E MÉTODOS}

Os experimentos foram conduzidos na área experimental da Fazenda de Ensino, Pesquisa e Extensão (FEPE) da FCA/UNESP - Câmpus de Botucatu. No ano agrícola 2009-2010, semeou-se a cultivar de soja FTS Campo Mourão RR, e BRS Valiosa RR no ano agrícola 2010-2011. Em ambos os anos agrícolas, a semeadura foi realizada no espaçamento de 0,45 m entre as linhas com densidade de 17 sementes $\mathrm{m}^{-1}$ conduzidas no sistema de semeadura direta. A área do cultivo da soja está situada a uma altitude de $724 \mathrm{~m}$, com as coordenadas geográficas de $22^{\circ} 48^{\prime} \mathrm{S}$ e $48^{\circ} 25^{\prime} \mathrm{O}$.

\section{Experimento 1: Depósitos da pulverização}

Avaliou-se o efeito da taxa de aplicação associado ou não ao uso do surfactante copolímero de poliéter e silicone $100 \% \mathrm{~m} \mathrm{v}^{-1}$ (Silwet L-77 AG), a $0,1 \%\left(\mathrm{v} \mathrm{v}^{-1}\right)$, sobre a deposição da pulverização nas plantas de soja. Os ensaios foram conduzidos no delineamento experimental em blocos ao acaso, no esquema fatorial $3 \times 2$ [três taxas de aplicação: $60 \mathrm{~L} \mathrm{ha}^{-1} \mathrm{com}$ ponta de pulverização (PP) modelo XR 8001, pressão na barra (PB) de $200 \mathrm{kPa} ; 110 \mathrm{~L} \mathrm{ha}^{-1}$ com PP modelo XR 8015, PB de $300 \mathrm{kPa}$ e $160 \mathrm{~L} \mathrm{ha}^{-1}$ com PP modelo XR 8002, PB de $400 \mathrm{kPa}$ ], com e sem surfactante, com quatro repetições. Todas as pontas produziram gotas finas de acordo com o fabricante (SPRAYING SYSTEMS, 2008).

O pulverizador utilizado foi o Advance Vortex 2000 (Jacto S.A.), com barra de 18,5 m de comprimento e pontas espaçadas de $0,50 \mathrm{~m}$, tracionado pelo trator Massey Fergunson 292, na velocidade (conjunto trator-pulverizador) de $1,86 \mathrm{~m} \mathrm{~s}^{-1}\left(6,7 \mathrm{~km} \mathrm{~h}^{-1}\right)$. A velocidade média do fluxo de ar ao longo da barra, medida a $0,50 \mathrm{~m}$ em relação à saída do ar, foi de $9,8 \mathrm{~m} \mathrm{~s}^{-1}$.

Pulverizações da calda contendo o corante marcador Azul Brilhante (FD\&C Blue n.1) foram realizadas por uma das secções da barra operando no sentido das linhas de plantio da cultura. As condições ambientais no momento da pulverização foram mensuradas a cada 20 minutos por termo-higrômetro (Lutron, modelo HT-3003/ precisão: $\pm 2 \%$ ) e anemômetro digital (Lutron, modelo AM - 4201/ precisão: $\pm 2 \%$ ). Leituras de UR e temperatura do ar foram mensuradas posicionando o termo-higrômetro a $1,0 \mathrm{~m}$ acima do solo na sombra e a velocidade do vento a $0,50 \mathrm{~m}$ acima do dossel da cultura.

Os depósitos da pulverização foram mensurados coletando-se três folhas por planta de dez plantas das linhas centrais de cada parcela $(8 \times 9 \mathrm{~m})$, nas partes superior (folhas do ponteiro), inferior (últimas folhas da parte inferior) e mediana (folhas posicionadas entre a parte superior e a inferior). Após a pulverização e secagem do corante, as folhas foram retiradas das plantas, colocadas em sacos de plástico e levadas para o laboratório, para a extração do marcador. A cada folha, adicionaram-se $30 \mathrm{~mL}$ de água destilada, agitando-as por $15 \mathrm{~s}$. Os depósitos foram quantificados em espectrofotômetro (Shimadzu UV 1601 PC, com comprimento de onda de $630 \mathrm{~nm}$ ).

Com a curva de calibração, obtida a partir de soluções-padrão, os dados de absorbância foram transformados em concentração $\left(\mathrm{mg} \mathrm{L}^{-1}\right) \mathrm{e}$, de posse da concentração inicial da calda e do volume de diluição das amostras, determinou-se a massa de corante retido na folha por meio da [eq. (1)]:

$$
\mathrm{Vi}=(\mathrm{Cf} \times \mathrm{Vf}) / \mathrm{Ci}
$$

em que,

$\mathrm{Ci}=$ concentração do corante na calda $\left(1.500 \mathrm{mg} \mathrm{L}^{-1}\right)$;

$\mathrm{Vi}=$ volume capturado pelo alvo $(\mathrm{mL})$;

$\mathrm{Cf}=$ concentração do corante detectada no espectrofotômetro em absorbância $\left(\mathrm{mg} \mathrm{L}^{-1}\right)$,

$\mathrm{Vf}=$ volume de diluição da amostra $(30 \mathrm{~mL})$. 
Após a extração, a área foliar foi determinada pelo medidor de área foliar de bancada LICOR (LI-3100). Para melhor apresentação dos dados, o volume retido em cada folha em $\mathrm{mL}$ foi transformado em $\mu \mathrm{L}$ e dividido pela sua respectiva área foliar obtendo-se, assim, a quantidade em $\mu \mathrm{L} \mathrm{cm}{ }^{-2}$. Os dados do depósito foram ajustados para que a quantidade de corante pulverizada nas parcelas fosse igual a $165 \mathrm{~g} \mathrm{ha}^{-1}$, tomando-se como referência a taxa de aplicação de $110 \mathrm{~L} \mathrm{ha}^{-1}$. Para equiparar a quantidade de $165 \mathrm{~g} \mathrm{ha}^{-1}$ do corante, foram multiplicados os valores dos depósitos encontrados nas parcelas que receberam a taxa de aplicação de $60 \mathrm{~L} \mathrm{ha}^{-1}$ pelo fator de correção 1,83 e, nas parcelas que receberam taxa de aplicação de $160 \mathrm{~L} \mathrm{ha}^{-1}$, o fator de 0,69 . Dessa forma, todas as parcelas, independentemente da taxa de aplicação, foram corrigidas para receber a mesma quantidade de corante.

Os valores dos depósitos da pulverização foram submetidos à análise de variância, no esquema fatorial $3 \times 2$ (taxa de aplicação x surfactante) e $3 \times 3$ (taxa de aplicação x parte de amostragem da planta) e, quando o teste $F$ indicou significância $(p<0,05$ ou $p<0,01)$, as médias foram comparadas pelo teste de Tukey $(\mathrm{p}<0,05)$.

\section{Experimento 2: Controle da ferrugem-asiática da soja (FAS)}

Para verificar os efeitos das tecnologias de aplicação no controle da FAS, os ensaios foram conduzidos no delineamento experimental em blocos ao acaso, com quatro repetições, no esquema fatorial $3 \times 2+1$ (tratamentos já utilizados com acréscimo de um tratamento sem aplicação de fungicida, testemunha). Os equipamentos e suas calibrações foram os mesmos descritos anteriormente.

A partir do estádio V8 da cultura, foram realizados levantamentos semanais para o monitoramento da infestação natural da FAS, observando visualmente os folíolos da parte inferior das plantas e, quando confirmados os primeiros sintomas da doença, procedeu-se à primeira aplicação da mistura fungicida piraclostrobina, associado com epoxiconazole (Opera®), na dosagem de $25+66,5 \mathrm{~g}$ i.a. $\mathrm{ha}^{-1}$. As demais aplicações foram realizadas com a mesma mistura fungicida, na mesma dosagem.

A avaliação da severidade da FAS foi realizada a intervalos de sete dias, atribuindo-se nota visual em quinze folíolos das partes inferior e mediana das plantas, utilizando a escala diagramática proposta por GODOY et al. (2006). As datas das avaliações da severidade da doença e os respectivos valores médios, nos trinta folíolos amostrados em cada parcela, foram usados para calcular a área abaixo da curva de progresso da doença (AACPD), conforme metodologia proposta por CAMPBELL \& MADDEN (1990), por meio da [eq. (2)]:

$$
\underset{i=1}{\mathrm{AACPD}}=\sum\left[\left(\mathrm{x}_{\mathrm{i}+1}+\mathrm{x}_{\mathrm{i}}\right) / 2\right]^{*}\left(\mathrm{t}_{\mathrm{i}+1}-\mathrm{t}_{\mathrm{i}}\right)
$$

em que,

$\mathrm{x}$ - severidade média da doença ferrugem-asiática na parcela experimental;

$\mathrm{x}_{\mathrm{i}}$ - severidade média da doença na parcela experimental no tempo $\mathrm{t}_{\mathrm{i}}$;

$\mathrm{n}$ - número de avaliações;

$\mathrm{t}$ - data da avaliação,

$\left(t_{i+1}-t_{i}\right)$ - intervalo de tempo (dias) entre duas avaliações consecutivas.

A produtividade foi medida colhendo-se as três linhas centrais de cada parcela, com 5,0 m de comprimento, por uma colhedora de parcelas (Wintersteiger Nursery Master Elite A-4910 Ried/Austria). Após a colheita, procedeu-se à pesagem dos grãos, corrigindo a umidade para $13 \%$ (b.u.) e os valores transformados em $\mathrm{kg} \mathrm{ha}^{-1}$. O peso de mil grãos (PMG) foi mensurado com a mesma umidade. 
Os valores da AACPD, PMG e produtividade foram submetidos à análise de variância $(3 \times 2+1)$ e quando o teste $F$ indicou significância $(p<0,05$ ou $p<0,01)$, as médias foram comparadas pelo teste de Tukey $(\mathrm{p}<0,05)$. Quando a interação entre fatorial $\mathrm{x}$ testemunha foi significativa pelo teste $\mathrm{F}$, todos os tratamentos foram comparados entre si e com a testemunha, pelo teste de Tukey $(\mathrm{p}<0,05) . \mathrm{O}$ coeficiente de correlação de Pearson $(\mathrm{p}<0,05)$ foi utilizado para avaliar a correlação entre AACPD e produtividade da soja. Todas as análises foram realizadas com o uso do programa ASSISTAT versão 7.7 (SILVA \& AZEVEDO, 2009).

\section{RESULTADOS E DISCUSSÃO}

\section{Experimento 1: Depósitos da pulverização}

Na aplicação do corante marcador, realizada em 02-02-2010, aos 62 dias após a emergência (DAE), no estádio R2 da cultura da soja, foram registrados UR do ar de $57 \% \pm 5$, temperatura de $32^{\circ} \mathrm{C} \pm 2$ e velocidade do vento (VV) variando de 0,55 a $1,11 \mathrm{~m} \mathrm{~s}^{-1}$. No dia $02-02-2011$, aos 72 DAE, no estádio R2, foram registrados UR do ar de $67 \% \pm 5 \%$, temperatura de $30{ }^{\circ} \mathrm{C} \pm 2{ }^{\circ} \mathrm{C}$ e VV variando de 0,69 a $1,44 \mathrm{~m} \mathrm{~s}^{-1}$.

Os valores médios dos depósitos da pulverização para as taxas de aplicação, na presença e ausência de surfactante nas partes superior, mediana e inferior das plantas de soja, no ano agrícola 2009-2010, são descritos na Tabela 1. Interações significativas ( $<<0,01 ; \mathrm{F}=11,6 ; \mathrm{CV}$ : 25\%) foram observadas apenas nas folhas da parte superior para a interação taxa de aplicação x surfactante (Tabela 1).

A taxa de aplicação de $60 \mathrm{~L} \mathrm{ha}^{-1}$ com surfactantes diferiu $(\mathrm{p}<0,05 ; \mathrm{F}=3,4)$ da taxa de $160 \mathrm{~L} \mathrm{ha}^{-1}$. A taxa de $110 \mathrm{~L} \mathrm{ha}^{-1}$ não diferiu das taxas de 60 e $160 \mathrm{~L} \mathrm{ha}^{-1}$, sendo o maior valor de depósito encontrado para a menor taxa aplicada. Na aplicação realizada sem surfactante, verificouse que a taxa de $60 \mathrm{~L} \mathrm{ha}^{-1}$ apresentou o menor valor de depósito, diferindo $(\mathrm{p}<0,01 ; \mathrm{F}=10,2)$ dos valores obtidos nas taxas de 110 e $160 \mathrm{~L} \mathrm{ha}^{-1}$ (Tabela 1). Uma possível explicação para os menores valores de depósitos encontrados, quando aplicadas as maiores taxas na presença de surfactante, seria a saturação da superfície foliar pelas gotas em decorrência da diminuição da tensão superficial, proporcionada pelo surfactante e, posteriormente, o escorrimento do produto aplicado. Esse escorrimento provavelmente se intensificou pelo aumento da taxa de aplicação com a diminuição dos depósitos, na medida em que aumentou a taxa de aplicação. IOST \& RAETANO (2010), estudando o comportamento de adjuvantes na redução da tensão superficial dinâmica e ângulo de contato com superfícies naturale artificial, observaram que os surfactantes organosiliconados foram os mais eficientes na redução da tensão superficial e proporcionaram maior molhamento de superfícies. SILVA et al. (2008) observaram que a adição de surfactante tensoativo à calda de pulverização reduziu a capacidade de retenção da calda em folhas de cafeeiro.

Tanto nas folhas da parte mediana $(\mathrm{CV}=42 \%)$ quanto da parte inferior das plantas de soja $(\mathrm{CV}=30 \%)$, não foram observadas diferenças entre as tecnologias estudadas sobre os depósitos da pulverização (Tabela 1).

Quando comparado à taxa de aplicação com as partes da planta, o teste foi significativo ( $<<0,01 ; \mathrm{F}=138,9 ; \mathrm{CV}=33 \%$ ) apenas para o efeito das partes da planta amostrada, indicando variação na distribuição dos depósitos da pulverização ao longo do dossel da cultura. Analisando os depósitos nas diferentes partes da planta, verificam-se maiores depósitos nas folhas da parte superior com $64 \%(0,442)$ da calda retida, seguida da mediana com $24 \%(0,167)$ e $12 \%(0,086)$ na parte inferior. Contrapondo os depósitos entre as diferentes taxas de aplicação, não foi observada diferença significativa entre os valores médios de depósitos da pulverização, independentemente da taxa aplicada $(\mathrm{p}=0,19 ; \mathrm{F}=1,71)$ (Tabela 1$)$. 
TABELA 1. Valores médios dos depósitos da pulverização nas partes superior, mediana e inferior das plantas de soja, nas taxas de aplicação de 60; 110 e $160 \mathrm{~L} \mathrm{ha}^{-1}$, com e sem surfactante no ano agrícola 2009-2010. Mean values of spray deposition level on upper, middle and bottom parts of soybean plants due to application rates of 60 , 110 and $160 \mathrm{~L} \mathrm{ha}^{-1}$ with and without silicone surfactant addition in the 2009-2010 crop season.

\begin{tabular}{|c|c|c|c|c|c|c|}
\hline \multirow{2}{*}{ Parte da planta } & \multirow{2}{*}{ Surfactante } & \multicolumn{3}{|c|}{ Taxa de aplicacão $\left(\mathrm{L} \mathrm{ha}^{-1}\right)$} & \multirow{2}{*}{\multicolumn{2}{|c|}{ Média }} \\
\hline & & 60 & 110 & 160 & & \\
\hline \multirow{2}{*}{ Superior } & Com & 0,555 a B & $0,440 \mathrm{ab} A$ & $0,353 \mathrm{~b} \mathrm{~A}$ & \multirow[b]{2}{*}{$0,442 \mathrm{~A}$} & dms surf.: 0,164 \\
\hline & Sem & $0,235 \mathrm{~b} \mathrm{~A}$ & 0,568 a $\mathrm{A}$ & 0,503 a $A$ & & dms taxa: 0,199 \\
\hline \multirow{2}{*}{ Mediana } & Com & 0,198 a $\mathrm{A}$ & 0,170 a $\mathrm{A}$ & 0,168 a $\mathrm{A}$ & \multirow{2}{*}{$0,167 \mathrm{~B}$} & dms surf.: 0,101 \\
\hline & Sem & 0,135 a $\mathrm{A}$ & 0,165 a $A$ & 0,168 a $\mathrm{A}$ & & $\mathrm{dms}$ taxa: 0,123 \\
\hline \multirow{2}{*}{ Inferior } & Com & 0,068 a A & 0,088 a $\mathrm{A}$ & 0,058 a $\mathrm{A}$ & \multirow{2}{*}{$0,086 \mathrm{C}$} & dms surf.: 0,039 \\
\hline & Sem & 0,075 a A & 0,065 a $A$ & 0,065 a $A$ & & dms taxa: 0,047 \\
\hline
\end{tabular}

Médias seguidas de mes ma letra, maiúscula na coluna e minúscula na linha, não diferem entre si, pelo teste de Tukey $(\mathrm{p}<0,05) / \mathrm{dms}$ : diferença mínima significativa/ dms entre médias da parte da planta: 0,054/ dms entre média da taxa de aplicação: 0,054

Os valores médios dos depósitos da pulverização para as taxas de aplicação, presença e ausência de surfactante nas partes superior, mediana e inferior das folhas de soja, no ano agrícola de 2010-2011, são descritos na Tabela 2. Não foram observadas diferenças nos valores médios dos depósitos nas folhas das partes superior $(\mathrm{CV}=42 \%)$, mediana $(\mathrm{CV}=66 \%)$ e inferior $(\mathrm{CV}=52 \%)$ entre taxas de aplicação na presença e ausência de surfactante siliconado, bem como na interação taxa de aplicação x surfactante.

Comparando a taxa de aplicação com as partes de amostragem das plantas, o teste foi significativo somente para variável taxa de aplicação $(\mathrm{p}<0,001 ; \mathrm{F}=71,8 ; \mathrm{CV}=61 \%)$. No ano agrícola de 2009-2010, valores de depósitos maiores foram observados nas folhas da parte superior com $74 \%(0,594)$ de retenção, seguida da parte mediana com $16 \%(0,129)$ e inferior com $10 \%(0,079)$. Variações da taxa de aplicação, como ocorrido no ano agrícola 2009/10, também não influenciaram a média dos valores dos depósitos ( $\mathrm{p}=0,75 ; \mathrm{F}=0,28)$ (Tabela 2$)$.

TABELA 2. Média dos valores dos depósitos da pulverização na parte inferior, mediana e inferior das plantas de soja nas taxas de aplicação de 60,110 e $160 \mathrm{~L} \mathrm{ha}^{-1}$ com e sem surfactante no ano agrícola de 2010-2011. Mean values of spray deposition level on upper, middle and bottom parts of soybean plants due to application rates of 60 , 110 and $160 \mathrm{~L} \mathrm{ha}^{-1}$ with and without silicone surfactant in the 2010-2011 crop season.

\begin{tabular}{|c|c|c|c|c|c|c|}
\hline \multirow{2}{*}{$\begin{array}{c}\text { Parte da } \\
\text { planta }\end{array}$} & \multirow[b]{2}{*}{ Surfactante } & \multicolumn{3}{|c|}{ Taxa de aplicação $\left(\mathrm{L} \mathrm{ha}^{-1}\right)$} & \multirow[b]{2}{*}{ Média } & \\
\hline & & 60 & 110 & 160 & & \\
\hline \multirow{2}{*}{ Superior } & Com & 0,760 a $\mathrm{A}$ & 0,520 a $\mathrm{A}$ & 0,795 a $\mathrm{A}$ & \multirow{2}{*}{$0,594 \mathrm{~A}$} & dms surf.: 0,371 \\
\hline & Sem & 0,505 a $\mathrm{A}$ & 0,530 a $\mathrm{A}$ & 0,453 a $A$ & & dms taxa: 0,451 \\
\hline \multirow{2}{*}{ Mediana } & Com & 0,100 a $\mathrm{A}$ & 0,098 a $\mathrm{A}$ & 0,123 a $\mathrm{A}$ & \multirow{2}{*}{$0,129 \mathrm{~B}$} & dms surf.: 0,125 \\
\hline & Sem & 0,108 a $\mathrm{A}$ & 0,158 a $\mathrm{A}$ & 0,188 a $\mathrm{A}$ & & dms taxa: 0,152 \\
\hline \multirow{2}{*}{ Inferior } & Com & 0,090 a $\mathrm{A}$ & 0,075 a $\mathrm{A}$ & 0,063 a $\mathrm{A}$ & \multirow{2}{*}{ 0,079 B } & dms surf.: 0,061 \\
\hline & Sem & 0,093 a A & 0,100 a $\mathrm{A}$ & 0,053 a $\mathrm{A}$ & & dms taxa: 0,074 \\
\hline Média & & $0,276 \mathrm{a}$ & $0,247 \mathrm{a}$ & $0,279 \mathrm{a}$ & & \\
\hline
\end{tabular}

Médias seguidas de mesma letra, maiúscula na coluna e minúscula na linha, não diferem entre si, pelo teste de Tukey $(\mathrm{p}<0,05) / \mathrm{dms}$ entre médias da parte da planta: 0,114/dms entre médias da taxa de aplicação: 0,114

Maiores valores dos depósitos foram obtidos nas folhas da parte superior, seguidos das partes medianas e inferiores, independentemente da taxa de aplicação, nos do is anos agrícolas estudados (Tabelas 1 e 2). Ficou evidente que as gotas da pulverização foram interceptadas pelas folhas da parte superior das plantas de soja e, consequentemente, depósitos maiores foram encontrados nessa 
região das plantas. Os resultados encontrados neste experimento corroboram aqueles obtidos por BARBOSA et al. (2009), que estudando as taxas de aplicação de 46,7; 93,4 e $140 \mathrm{~L} \mathrm{ha}^{-1}$, não observaram diferenças nos depósitos da pulverização nas folhas da parte inferior das plantas de soja, em que o aumento da taxa de aplicação não propiciou melhorias na deposição, nessa região da planta.

O aumento da taxa de aplicação de 60 para $160 \mathrm{~L} \mathrm{ha}^{-1}$, teoricamente, não implicaria melhorias no controle de doenças que atacam as partes inferiores da planta de soja como a FAS, uma vez que essa doença se inicia na parte inferior das plantas. Esta parte do dossel propicia um microclima favorável à doença. A redução da taxa de aplicação, desde que não prejudique a eficiência de controle, torna-se alternativa interessante, pois permite economia de água, aumento da capacidade operacional dos pulverizadores e redução dos custos operacionais.

O uso do surfactante siliconado não resultou em incrementos nos depósitos da pulverização no dossel da cultura da soja, nos dois anos agrícolas. CUNHA \& PERES (2010), avaliando o efeito do adjuvante dodecil benzeno combinado com $\mathrm{N}$ e $\mathrm{P}_{2} \mathrm{O}_{5}$, obtiveram aumento de densidade de gotas nas folhas das partes superior e mediana da planta, na presença do surfactante, porém não observaram melhorias na cobertura nas folhas da parte inferior das plantas. AGUIAR-JÚNIOR et al. (2011), estudando o efeito combinado da assistência de ar em barra de pulverização com e sem adjuvantes, não observaram diferença nos níveis dos depósitos da pulverização, quando foi adicionado o surfactante copolímero de poliéter e silicone à calda de pulverização.

A menor quantidade de calda retida nas folhas da parte inferior das plantas é uma característica não desejada, quando o fungicida é recomendado para o controle de doenças que se iniciam nessa parte das plantas. Alternativas visando ao aumento dos depósitos da pulverização devem ser estudadas, uma vez que o aumento da taxa de aplicação, como apresentado neste trabalho e por BARBOSA et al. (2009), e o uso do surfactante siliconado não foram suficientes ou não apresentaram correlação com maior penetração das gotas no dosse l da cultura da soja.

\section{Experimento 2: Controle da ferrugem-asiática da soja (FAS)}

As condições meteorológicas e do estádio de desenvolvimento da cultura, no momento das aplicações dos fungicidas, estão descritas na Tabela 3.

TABELA 3. Condições meteorológicas e estádios de desenvolvimento da soja durante as aplicações fungicidas. Environmental conditions and growing stages during fungicide applications.

\begin{tabular}{cccccc}
\hline Data & $\begin{array}{c}\text { Dias após a } \\
\text { emergência }\end{array}$ & $\begin{array}{c}\text { Estádio da } \\
\text { cultura }\end{array}$ & $\begin{array}{c}\text { Umidade } \\
\text { relativa } \\
\text { do } \operatorname{ar}(\%)\end{array}$ & $\begin{array}{c}\text { Temperatura } \\
\text { do ar }\left({ }^{\circ} \mathrm{C}\right)\end{array}$ & Velocidade do vento $\left(\mathrm{m} \mathrm{s}^{-1}\right)$ \\
\hline 01 fev-2010 & 61 & R2 & $60 \pm 5$ & $31 \pm 2$ & $0,5-1,3$ \\
10 fev-2010 & 70 & R3 & $83 \pm 5$ & $26 \pm 2$ & $0,5-1,4$ \\
03 mar-2010 & 98 & R5 & $70 \pm 5$ & $26 \pm 3$ & $1,9-3,1$ \\
21 jan-2011 & 60 & R2 & $57 \pm 5$ & $31 \pm 2$ & $0,7-2,2$ \\
17 fev-2011 & 87 & R4 & $62 \pm 5$ & $30 \pm 3$ & $0,3-1,0$ \\
\hline
\end{tabular}

Resultados da análise de variância dos valores médios de área abaixo da curva de progresso da doença (AACPD), de peso de mil grãos (PMG) e produtividade da soja, nos anos agrícolas de 2009-2010 e 2010-2011 estão apresentados na Tabela 4. 
TABELA 4. Resultados da análise de variância para os valores médios de área abaixo da curva de progresso da doença (AACPD), de peso de mil grãos (PMG) e de produtividade da soja. Variance analysis results of area under the disease-progress curve (AUDPC), thousand-grain weight (TGW) and soybean yield.

\begin{tabular}{|c|c|c|c|c|c|c|}
\hline \multirow{3}{*}{ Causa de variação } & \multicolumn{6}{|c|}{ Valores de F } \\
\hline & \multicolumn{2}{|c|}{ AACPD } & \multicolumn{2}{|c|}{ PMG } & \multicolumn{2}{|c|}{ Produtividade } \\
\hline & $2009-2010$ & $2010-2011$ & $2009-2010$ & $2010-2011$ & $2009-2010$ & $2010-2011$ \\
\hline Testemunha $\mathrm{x}$ Fatorial & $390,29 * *$ & $7,85^{*}$ & $177,64 * *$ & $2,04^{\mathrm{ns}}$ & $17,21 * *$ & $10,46^{* *}$ \\
\hline Taxa de aplicação (T) & $1,43^{\mathrm{ns}}$ & $4,56 *$ & $4,52 *$ & $0,10^{\mathrm{ns}}$ & $0,15^{\mathrm{ns}}$ & $6,12 * *$ \\
\hline Surfactante $(\mathrm{S})$ & $0,01^{\mathrm{ns}}$ & $4,75^{*}$ & $0,59^{\mathrm{ns}}$ & $0,24^{\mathrm{ns}}$ & $0,21^{\mathrm{ns}}$ & $0,63^{\mathrm{ns}}$ \\
\hline $\mathrm{T} \times \mathrm{S}$ & $0,98^{\mathrm{ns}}$ & $4,53 *$ & $0,62^{\mathrm{ns}}$ & $1,78^{\mathrm{ns}}$ & $0,14^{\mathrm{ns}}$ & $0,38^{\mathrm{ns}}$ \\
\hline$\overline{\mathrm{CV}(\%)}$ & 13,67 & 16,43 & 2,91 & 6,77 & 12,04 & 13,01 \\
\hline
\end{tabular}

*Significativo (p<0,01); **Significativo ( $\mathrm{p}<0,05) ;{ }^{\text {ns }} \mathrm{Não}$ significativo.

No ano agrícola de 2009-2010, todos os tratamentos que receberam aplicação de fungicida, independentemente da tecnologia utilizada, não diferiram $(\mathrm{CV}=11 \% ; \mathrm{F}=105,5 ; \mathrm{p}<0,01)$ entre si, entretanto todos os tratamentos diferiram da testemunha. No ano agrícola de 2010-2011 somente a taxa de $160 \mathrm{~L} \mathrm{ha}^{-1}$ com adição do surfactante siliconado diferiu (CV: 16\%; F: 5,1; p<0,01) do tratamento-testemunha. Maiores valores de AACPD (tratamentos que receberam aplicação de fungicida) foram constatados no ano agrícola de 2010-2011, comparados com o ano anterior (Figura 1). Evidencia-se a existência de uma relação entre severidade da FAS representada pela AACPD e tratamentos que receberam ou não aplicação fungicida. A diferença menor entre valores de AACPD dos tratamentos e testemunha, no ano agrícola de 2010-2011, implica menor eficiência de controle dos tratamentos fungicidas. Isso pode ser atribuído ao maior porte da planta (BRS Valiosa RR com altura superior a $1,4 \mathrm{~m}$ ), maior intensidade de chuvas na fase com maior predisposição a FAS (Dados não apresentados) e fatores intrínsecos à cultivar (cv.), quando comparada à cv. do ano agrícola anterior (FTS Campo Mourão RR) com altura média de 1,0 m (Figura 1).

A maior taxa de aplicação na presença do surfactante, provavelmente, proporcionou maiores quantidades de depósitos de fungicida nas partes mediana e inferior das plantas de soja, embora essa diferença não seja evidenciada nos ensaios de depósitos da pulverização (Experimento 1).
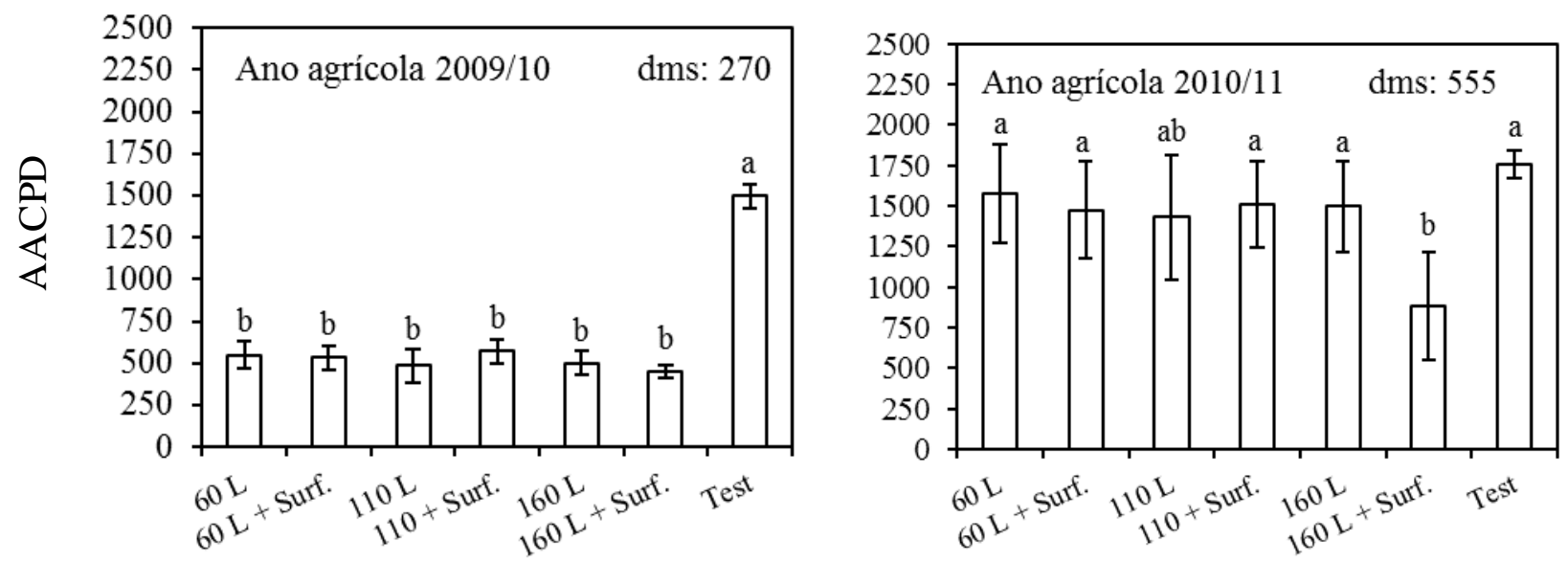

FIGURA 1. Severidade média da ferrugem-asiática da soja (FAS) representada pelos valores da área abaixo da curva de progresso da doença (AACPD). (Desvio-padrão da média está representado pelas linhas verticais). Médias com mesma letra não diferem entre si, pelo teste de Tukey $(\mathrm{p}<0,05)$. Ave rages of Asian soybean rust (ASR) severity represented by values of area under the disease-progress curve (AUDPC). (The error bars represent standard deviation of means). Means with the same letter did not differ by the Tukey test $(\mathbf{p}<0.05)$. 
Os valores médios de AACPD, em função da taxa de aplicação, no ano agrícola de 2010-2011, são apresentados na Figura 2. A aplicação do fungicida na taxa de $160 \mathrm{~L} \mathrm{ha}^{-1}$ apresentou valores menores de AACPD, enquanto nos tratamentos com taxa de $60 \mathrm{~L} \mathrm{ha}^{-1}$ encontram-se os valores maiores. Valores intermediários de AACPD foram obtidos com a taxa de $110 \mathrm{~L} \mathrm{ha}^{-1}$.

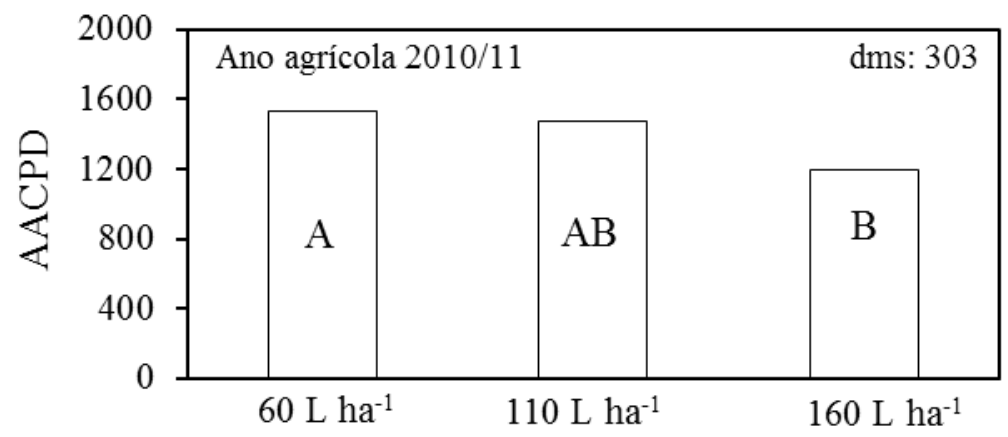

FIGURA 2. Efeito da taxa de aplicação na severidade da doença representada pelos valores de área abaixo da curva de progresso da doença (AACPD). Médias com mesma letra não diferem entre $s i$, pelo teste de Tukey $(\mathrm{p}<0,05)$. Application rate effect on disease severity represented by values of area under the disease-progress curve (AUDPC). Means with the same letter did not differ by the Tukey test $(\mathbf{p}<0.05)$.

ZYL et al. (2010), estudando o efeito de diferentes adjuvantes associados a fungicidas no controle de Botrytis cinerea, em folhas de videira, demonstraram o potencial dos adjuvantes pertencentes à classe dos organossiliconados em aumentar a bioeficácia do fungicida. Algumas propriedades dos adjuvantes em folhas de feijão, batata e cebola foram avaliadas por GENT et al. (2003). Os autores constataram que o surfactante organossiliconado $\left(0,125 \% \mathrm{v} \mathrm{v}^{-1}\right)$ proporcionou melhor cobertura da calda pulverizada sobre as folhas dessas culturas e maior absorção de fungicidas pertencentes ao grupo dos triazóis, na presença desse adjuvante, embora a taxa de absorção fosse variável com o tipo de cultura.

No presente trabalho, o uso do surfactante proporcionou efeito positivo na eficiência de controle da FAS com maior taxa de aplicação, no ano agrícola de 2010-2011 (Tabela 5). Valores menores de AACPD, neste estudo, podem estar relacionados às propriedades que os surfactantes siliconados podem proporcionar à calda de pulverização, como maior espalhamento da calda pulverizada, maior retenção do ingrediente ativo e facilitador da absorção, e translocação do agroquímico pelas folhas (HOLLOWAY et al., 2000).

O desdobramento dos valores médios da AACPD para a interação taxa de aplicação $\mathrm{x}$ surfactante, no ano agrícola de 2010-2011 está representado na Tabela 5. Diferenças entre aplicações com e sem surfactante foram encontradas apenas quando se utilizou a taxa de $160 \mathrm{~L} \mathrm{ha}^{-1}$, com valor méd io menor da AACPD detectado na presença do surfactante.

TABELA 5. Desdobramento dos valores médios da área abaixo da curva de progresso da doença (AACPD) para a interação taxa de aplicação x surfactante, no ano agrícola de 20102011. Breakdown of means of area under the disease-progress curve (AUDPC) for the interaction between application rates and surfactant use in the 2010-2011 crop season.

\begin{tabular}{lccc}
\hline \multirow{2}{*}{ Surfactante siliconado } & \multicolumn{3}{c}{ Taxa de aplicação $\left(\mathrm{L} \mathrm{ha}^{-1}\right)$} \\
\cline { 2 - 4 } & 60 & 110 & 160 \\
\hline Sem & $1575,8 \mathrm{~A} \mathrm{a}$ & $1430,2 \mathrm{~A} \mathrm{a}$ & $1496,6 \mathrm{~A} \mathrm{a}$ \\
Com & $1476,9 \mathrm{~A} \mathrm{a}$ & $1506,7 \mathrm{~A} \mathrm{a}$ & $884,5 \mathrm{~B} \mathrm{~b}$ \\
\hline
\end{tabular}

Médias seguidas de mesma letra, maiúscula na coluna e minúscula na linha, não diferem entre si, pelo teste de Tukey $(\mathrm{p}<0,05) / \mathrm{dms}$ (coluna): 353/ dms (linha): 429 
A redução nos valores de AACPD é considerada um fator importante no controle da FAS, pois existem correlações negativas entre AACPD e produtividade da soja (MUELLER et al., 2009; PRADO et al., 2010; TWIZEYIMANA et al, 2011). O uso de surfactante siliconado na calda fungicida pode ser uma alternativa viável na melhoria do controle químico da FAS, uma vez que a adição desse produto à calda fungicid a proporcionou redução na intensidade da doença.

No ano agrícola de 2009-2010 os tratamentos que receberam aplicação de fungicidas apresentaram valores médios de $\mathrm{PMG}$ superiores $(\mathrm{CV}=4 \% ; \mathrm{F}=16,7 ; \mathrm{p}<0,01)$ àqueles da testemunha, no entanto não diferiram entre si. No ano agrícola seguinte, não houve diferenças ( $\mathrm{CV}=7 \% ; \mathrm{F}=1,6$; p>0,05) entre os tratamentos, quando a valiado o PMG (Figura 3 ).
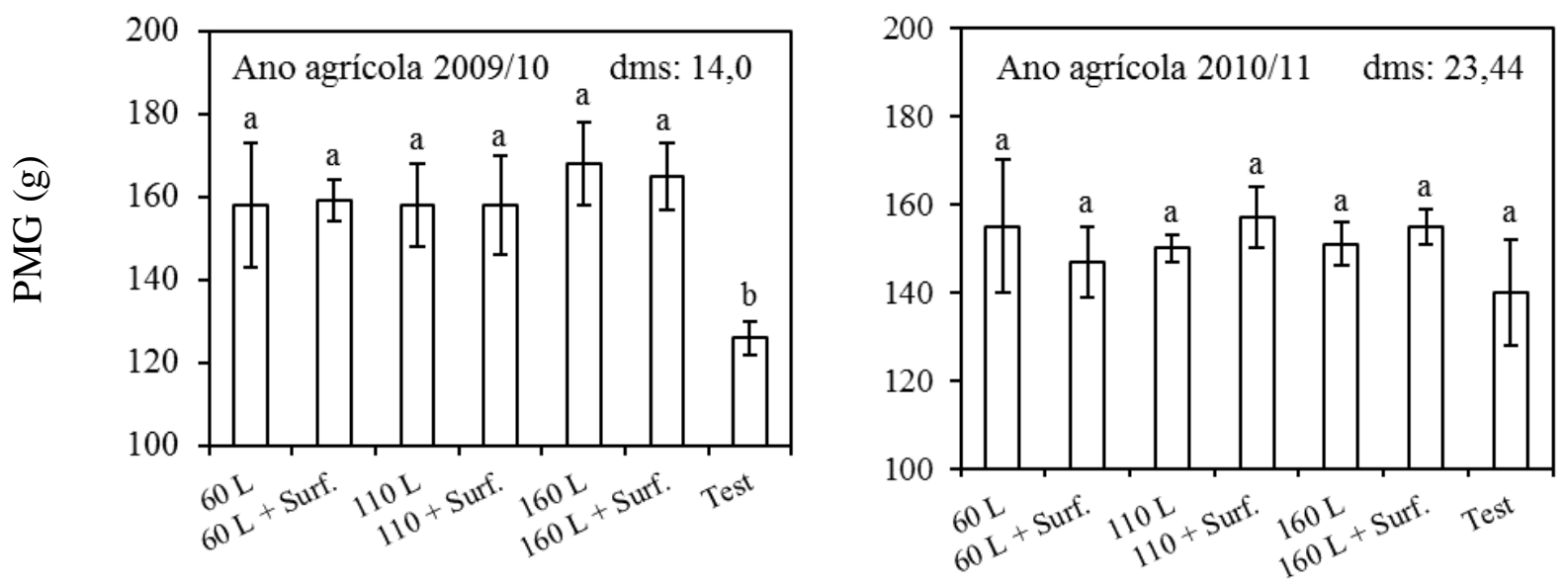

FIGURA 3. Valores médios do peso de mil grãos (PMG) da soja. (Des vio-padrão da média está representado pelas linhas verticais). Médias com mesma letra não diferem entre si, pelo teste de Tukey $(\mathrm{p}<0,05)$. Thousand-grain weight (TGW) mean values (The error bars represent standard deviation of means). Means with the same letter did not differ by the Tukey test $(p<0.05)$.

Todos os tratamentos que receberam aplicação de fungicida diferiram $(\mathrm{CV}=8 \% ; \mathrm{F}=5,9$; $\mathrm{p}<0,01)$ da testemunha, no ano agrícola de 2009-2010, e os tratamentos que receberam pulverizações não diferiram entre si. No ano agrícola de 2010-2011, os tratamentos com maiores taxas de aplicação (160 $\left.\mathrm{L} \mathrm{ha}^{-1}\right)$, com e sem adição do surfactante siliconado, apresentaram valores médios de produtividade maiores, sendo os únicos a diferirem da testemunha $(\mathrm{CV}=14 \% ; \mathrm{F}=3,6$; $\mathrm{p}<0,05)$.
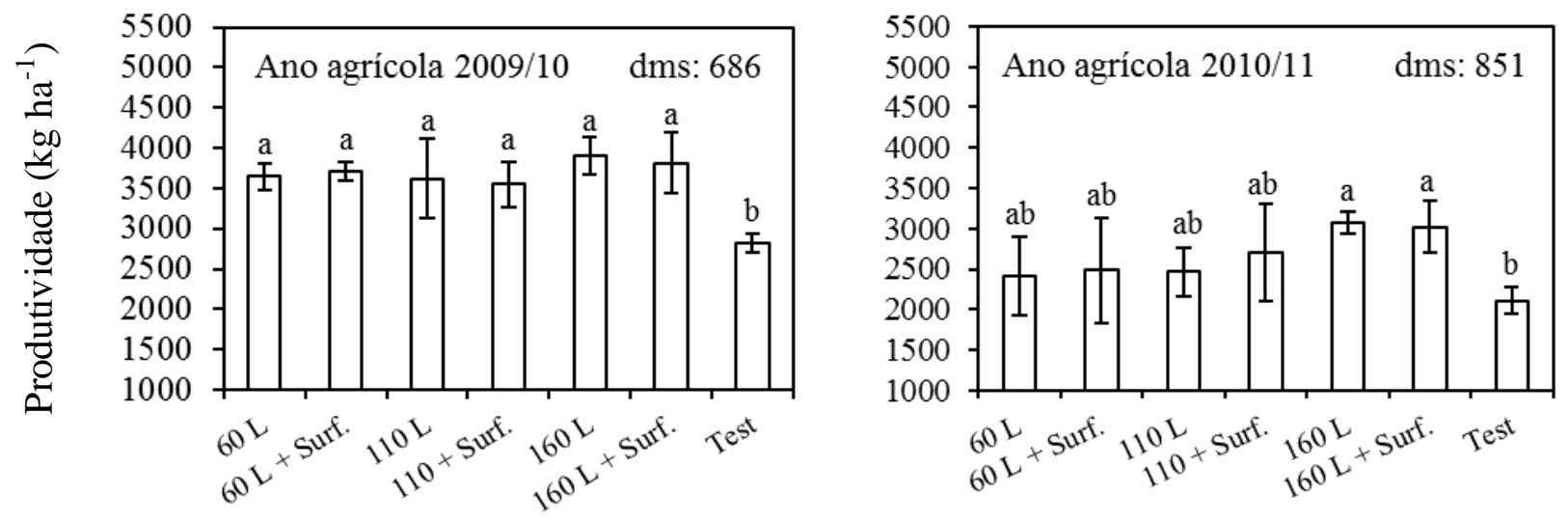

FIGURA 4. Valores médios de produtividade da soja. (Desvio-padrão da média está representado pelas linhas verticais). Médias com mesma letra não diferem entre si, pelo teste de Tukey $(\mathrm{p}<0,05)$. Soybean yield means (The error bars represent standard deviation of means). Means with the same letter did not differ by the Tukey test $(\mathbf{p}<\mathbf{0 . 0 5})$. 
O maior valor médio de produtividade foi encontrado no tratamento que recebeu a taxa de aplicação de $160 \mathrm{~L} \mathrm{ha}^{-1}$, diferindo daqueles com taxas de aplicação menores (Figura 5). A aplicação na taxa de $160 \mathrm{~L} \mathrm{ha}^{-1}$ constitui a melhor opção no controle da FAS com fungicidas, reduzindo as perdas causadas por essa doença e proporcionando incremento na produtividade, de aproximadamente $18 \%$ em relação às taxas de aplicação de 60 e $110 \mathrm{~L} \mathrm{ha}^{-1}$.

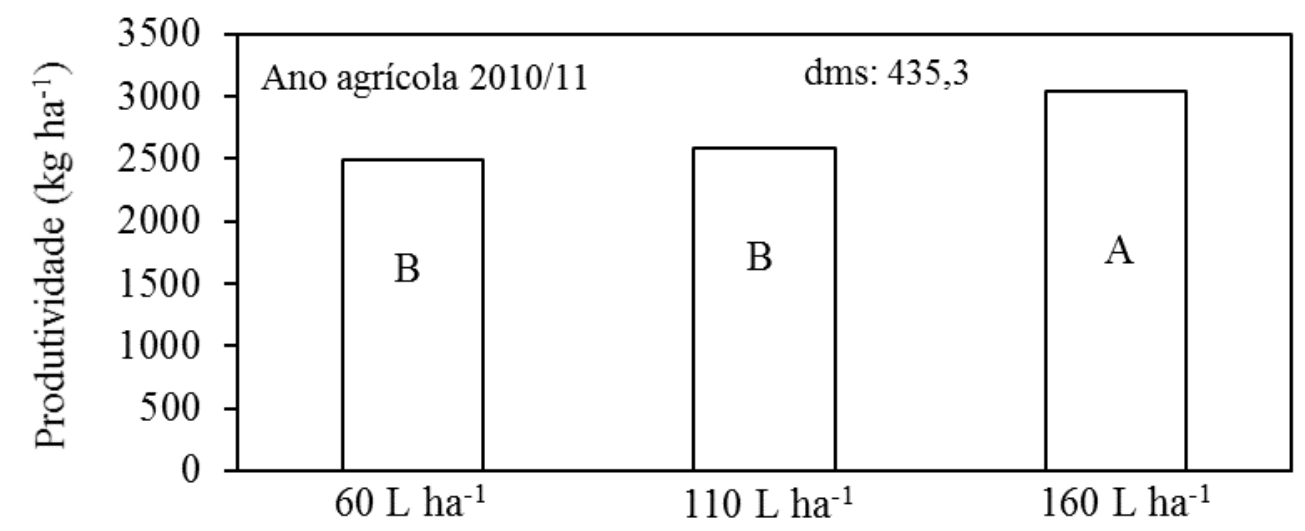

FIGURA 5. Efeito da taxa de aplicação na produtividade da soja, no ano agrícola de 2010-2011. Médias com mesma letra não diferem entre si, pelo teste de Tukey $(p<0,05)$. Application rate effects on soybean yield in the 2010-2011 crop season. Means with the same letter did not differ by the Tukey test $(p<0.05)$.

Correlações negativas significativas entre produtividade e AACPD ocorreram nos dois anos agrícolas estudados $(\mathrm{p}<0,01)$. Observou-se correlação negativa moderada no ano agrícola de 20092010 ( $\mathrm{r}=-0,65 ; \mathrm{F}=19,06)$ e no ano agrícola de 2010-2011 ( $\mathrm{r}=-0,55 ; \mathrm{F}=11,47)$ (Figura 6). Quanto maior a severidade da doença (AACPD), menor a produtividade da cultura, em ambos os anos agrícolas. Considerando que a severidade da doença influencia de forma significativa a capacidade produtiva das plantas de soja, o controle da FAS torna-se prática indispensável, visando à diminuição das perdas de produtividade. O controle químico com fungicidas, na taxa de aplicação adequada ao estádio de desenvolvimento da planta, torna-se indispensável como forma de garantia da produtividade na cultura da soja.

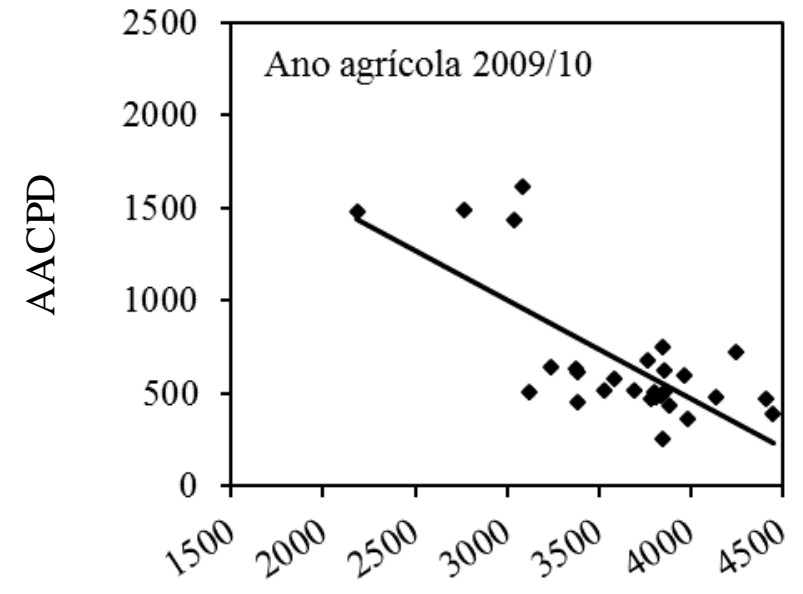

Produtividade $\left(\mathrm{kg} \mathrm{ha}^{-1}\right)$

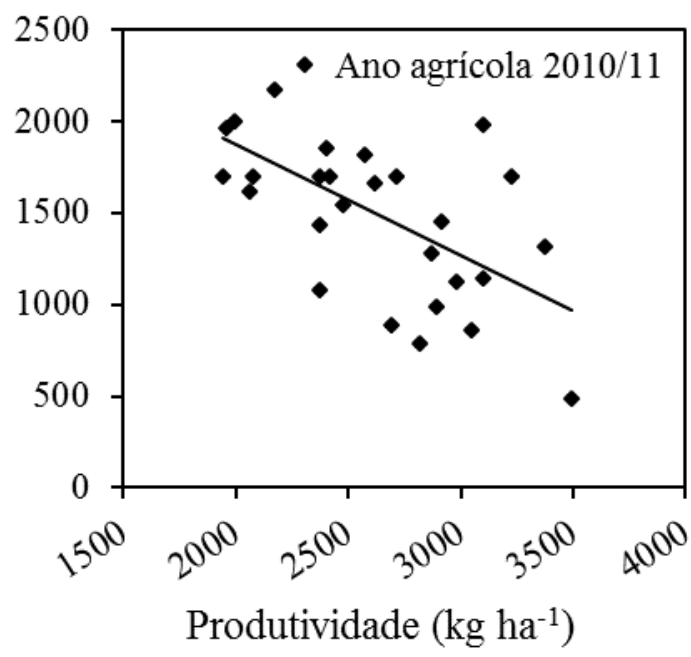

FIGURA 6. Correlação entre a severidade da ferrugem-asiática da soja (FAS) representada pelos valores da área abaixo da curva de progresso da doença (AACPD) e produtividade. Correlation between Asian soybean rust (ASR) disease severity and soybean yield represented by values of area under the disease-progress curve (AUDPC). 


\section{CONCLUSÕES}

As taxas de aplicação de 60, 110 e $160 \mathrm{~L} \mathrm{ha}^{-1}$ e o uso do surfactante siliconado não influenciaram nos depósitos da pulverização, nas folhas das partes mediana e inferior das plantas de soja.

Maiores valores de depósitos da pulverização foram encontrados nas folhas da parte superior, seguidas da mediana e inferior.

O aumento na taxa de aplicação não proporcionou aumento de deposição nas folhas das plantas de soja.

A aplicação na taxa de $160 \mathrm{~L} \mathrm{ha}^{-1}$ com adição do surfactante à base de silicone à calda contribuiu para a redução da severidade da ferrugem-asiática na cultura da soja, no ano agrícola de 2010-2011.

A taxa de aplicação de $160 \mathrm{~L} \mathrm{ha}^{-1}$ proporcionou maior valor de produtividade da soja, no ano agrícola de 2010-2011.

\section{REFERÊNCIAS}

AGUIAR-JÚNIOR, H.O.; RAETANO, C.G.; PRADO, E.P.; DAL POGETTO, M.H.F.A.; CRISTOVAM, R.S.; GIMENES, M.J. Adjuvantes e assistência de ar em pulverizador de barras sobre a deposição da calda e controle de Phakopsora pachyrhizi (Sydow \& Sydow). Summa Phytopathologica, Botucatu, v.37, n.3, p.103-109, 2011.

ARMSTRONG-CHO, C. WOLF, T.; CHONGO, G.; GAN, Y.; HOGG, T.; LAFOND, G.; JOHNSON, E.; BANNIZA, S. The effect of carrier volume on ascochyta blight (Ascochyta rabiei) control on chickpea. Crop Protection, Amsterdam, v.27, p.1020-1030, 2008.

BARBOSA, R.N.; GRIFFIN, J.L.; HOLLIER, C.A. Effect of spray rate and method of application in spray deposition. Applied Engineering in Agriculture, Saint Joseph, v.25, n.2, p.181-184, 2009. (Technical Note).

BOLLER, W.; MACHRY, M. Efeito da pressão de trabalho e de modelos de pontas de pulverização sobre a eficiência de herbicida de contato em soja. Engenharia Agrícola, Jaboticabal, v.27, n.3, p.722-727, 2007.

CALVO E.S.; KIIHL, R.A.S.; GARCIA, A.; HARANDA, A.; HIROMOTO, M. Two major recessive soybean genes conferring soybean rust resistance. Crop Science, Madison, v.48, p.13501354, 2008.

CAMPBELL, C.L.; MADDEN, L.V. Introduction to plant disease epidemiology. New York: John Wiley e Sons, 1990. 532p.

CUNHA, J.P.A.R.; MOURA, E.A.C.; SILVA JÚNIOR, J.L.; ZAGO, F.A.; JULIATTI, F.C. Efeitos de pontas de pulverização no controle químico da ferrugem da soja. Engenharia Agrícola, Jaboticabal, v.28, n.2, p.283-291, 2008.

CUNHA, J.P.A.R.; PERES, T.C.M. Influência de pontas de pulverização e adjuvante no controle químico da ferrugem asiática da soja. Acta Scientiarum. Agronomy, Maringá, v.32, n.4, p.597$602,2010$.

CUNHA, J.P.R.; SILVA JUNIOR, A. D. Volumes de calda e pontas de pulverização no controle químico de Spodoptera frugiperda na cultura do sorgo forrageiro. Engenharia Agrícola, Jaboticabal, v.30, n.4, p.692-699, 2010.FURLAN, S.H. Impacto da ferrugem asiática da soja no Brasil. Summa Phytopathologica, Botucatu, v.31, n.1, p.119-120, 2005.

FURLAN, S.H. Impacto da ferrugem asiática da soja no Brasil. Summa Phytopathologica, Botucatu, v.31, n.1, p.119-120, 2005. 
GENT, D.H.; SCHW ARTZ, H.F.; NISSEN, S.J. Effect of commercial adjuvants on vegetable crop fungicide coverage, absorption, and efficacy. Plant Disease, Saint Paul, v.87, n.5, p.591-597, 2003.

GODOY, C.V.; KOGA, L.J.; CANTERI, M.G. Diagrammatic scale for assessment of soybean rust severity. Fitopatologia B rasileira, Brasília DF, v.31, n.1, p.63-68, 2006.

GOELlNER, K. LOEHRER, M., LANGENBACH, C.; CONRATH, U.; KOCH, E.; SCHAFFRATH, U. Phakopsora pachyrhizi, the causal agent of Asian soybean rust. Molecular Plant Pathology, Oxford, v.11, n.2, p.169-177, 2010.

HOLlOWAY, P.J., BUTLER ELLIS, M.C., WEBB, D.A., WESTERN, N.M., TUCK, C.R., HAYES, A.L., MILLER, P.C.H.: Effects of some agricultural tank-mix adjuvants on the deposition efficiency of aqueous sprays on foliage. Crop Protection, Amsterdam, v.19, p.27-37, 2000.

IOST, C.A.R.; RAETANO, C.G. Tensão superficial dinâmica e ângulo de contato de soluções aquosas com surfatantes em superfícies artificiais e naturais. Engenharia Agrícola, Jaboticabal, v.30, n.4, p.670-680, 2010.

LEVY, C. Epidemiology and chemical control of soybean rust in southern Africa. Plant Disease, Saint Paul, v.89, p.669-674, 2005.

MUELLER, T.A. MILES, M.R.; MOREL, W.; MAROIS, J.J.; WRIGHT, D.L.; KEMERAIT, R.C.; LEVY, C. HARTMAN, G.L. Effect of fungicides and timing of application on soybean rust severity and yield. Plant Disease, Saint Paul, v.93, n.2, p.243-248, 2009.

PRADO, E.P.; RAETANO, C.G.; AGUIAR-JÚNIOR, H.O.; DAL POGETTO, M.H.F.A.; CHRISTOVAM, R.S.; GIMENES, M.J.; ARAUJO, D. Velocidade do ar em barra de pulverização na deposição da calda fungicida, severidade da ferrugem asiática e produtividade da soja. Summa Phytopathologica, Botucatu, v.36, n.1, p.45-50, 2010.

RYCKAERT, B. SPANOGHE, P.; HAESAERT, G.; HEREMANS, B.; ISEBAERT, S.; STEURBAUT, W. Quantitative determination of the influence of adjuvants on foliar fungicide residues. Crop Protection, Amsterdam, v.26, p.1589-1594, 2007.

SILVA, A.R.; LEITE, M.T.; FERREIRA, M.C. Estimativa da área foliar e capacidade de retenção de calda fitossanitária em cafeeiro. Bioscience Journal, Uberlândia, v.24, n.3, p.66-73, 2008.

SILVA, F.A.S. E.; AZEVEDO, C.A. V. de. Principal components analysis in the software assistat-statistical attendance. In: WORLD CONGRESS ON COMPUTERS IN AGRICULTURE, 7; 2009, Reno-NV-USA: American Society of Agricultural and Biological Engineers, 2009.

SPRAYING SYSTEMS. Teejet spray products: catálago 50A-P. Wheaton: Spraying Systems, 2008, 192p.

TWIZEYIMANA, M.; OJIAMBO, P.S.; HARTMAN, G.L.; BANDYOPADHYAY, R. Dynamics of soybean rust epidemics in sequential plantings of soybean cultivars in Nigeria. Plant Disease, Saint Paul, v.95, p.43-50, 2011.

WALKER D.R.; BOERMA, H.R.; PHILLIPS, D.V.; SCHNEIDER, R.W.; BUCKLEY, J.B.;.SHIPE, E.R.; MUELLER, J.D.; WEAVER, D.B.; SIKORA, E.J.; MOORE, S.H.; HARTMAN, G.L.; MILES, M.R.; HARRIS, D.K.; WRIGHT, D.L.; MAROIS, J.J. Evaluation of USDA soybean germplasm accessions for resistance to soybean rust in the southern United States. Crop Science, Madison, v.51, n.678-693, 2011.

ZHU, H.; OZKAN, H.E.; REDING, M.E.; KRAUSE, C.R.; DERKSEN, R.C. Development of a canopy opener to improve spray deposition and coverage inside soybean canopies: Part 2. Opener design with field experiments. Transaction of the ASABE, Saint Joseph, v.51, n.6, p.1905-1912, 2008. 
ZABKIEWICZ, J.A. A cleaner and greener New Zealand thanks to 2,4,5-T, science and silicones. Che mistry in Ne w Zealand, Hamilton, p.8-12, 2008.

ZYL, S.A.V.; BRINK, J.; CALITZ, F.J.; COERTZE, S. FOURIE, P.H. The use of adjuvants to improve spray deposition and Botrytis cinerea control on Chardonnay grapevine leaves. Crop Protection, Amsterdam, v.29, p.58-67, 2010. 